\title{
The COVID-19 Pandemic: A Challenge for Obesity Research and Management
}

\author{
Hans Hauner \\ Else Kröner-Fresenius Center for Nutritional Medicine, Technical University of Munich, \\ Munich, Germany
}

The currently escalating COVID-19 pandemic has disrupted not only healthcare systems, but also many social and economic activities around the world. As a consequence, daily life has changed dramatically over the past few months, and many of these changes will probably be long-lasting. Without any doubt, we are still menaced by the spreading of SARS CoV-2, and we still understand little about its transmission, its clinical impact and, especially, the pathophysiology of its severe complications. To make matters worse, effective antiviral medication is not yet available, vaccinations are still in the trial phases, and it is still an open question as to when one will be ready for the general public.

But how much do we need to be concerned about COVID-19 in the obesity community? The answer to this simple question is clear and undisputable: we have to deal with COVID-19 and the more the better. The rationale for this policy is presented in the new Position Statement of the Obesity Management Task Force of EASO, the European Association for the Study of Obesity, published in this issue of Obesity Facts [1], which follows up on their previous statement on COVID-19 earlier this year [2]. This new comprehensive paper sheds light on this dominating health threat from a variety of perspectives, resulting in the unequivocal message that this virus represents an exceptional challenge for both obesity research and clinical management.

Against the background of numerous observational data, there is no doubt that obesity is a risk factor for developing COVID-19 and is associated with more severe disease progression and poorer survival, as people with obesity are at increased risk of exacerbation from acute viral respiratory infections. In this situation, they are more frequently admitted to intensive care unit (ICU) treatment, including longer duration of mechanical ventilation and longer duration of ICU care in general. One of the main explanations is that obesity is characterized by a decrease in pulmonary compliance, partly due to fat accumulation in the chest and the abdomen. The ventilatory reserve is reduced with a higher propensity to respiratory failure 
Hauner: The COVID-19 Pandemic

[3]. This goes along with an impaired immune function, as multiple disturbances of the innate and adaptive immune system have been described in people with obesity [3].

The toll to pay is high. A recent hospital survey from the UK revealed an increased risk of death (HR 1.33) linked to clinician-reported obesity in a large group of hospitalised patients [4]. This increased mortality risk may depend on the degree of obesity [5]. In addition and interesting to note is that obesity affects the risk of COVID-19 hospitalisation particularly in those under the age of 60 years [6].

Despite the close direct link between obesity and COVID-19, there is increased attention on how dramatically this pandemic has changed clinical care of patients with common noncommunicable diseases including obesity. This "collateral damage" caused by the pandemic is substantial. A quick survey of the ongoing clinical activities among the EASO-accredited Centers of Obesity Management (COM) across Europe revealed a marked reduction or even closure of obesity services, including surgical procedures for patients with severe obesity. Therefore, the recent initiative taken by the Diabetes Surgery Summit is very helpful to avoid further disadvantages for this particular patient group [7].

Thus, patients with obesity pose a specific and unprecedented challenge for healthcare in the COVID-19 pandemic, and the call for actions with many practical recommendations released by EASO is a valuable and welcome initiative for healthcare providers and other stakeholders across Europe and deserves full support and dissemination by all of us.

\section{Conflict of Interest Statement}

The author has no conflict of interest to declare.

\section{Funding Sources}

The author received no funding for this Editorial.

\section{References}

1 Dicker D, Bettini S, Farpour-Lambert N, Frühbeck G, Golan R, Goossens G, et al. Obesity and COVID-19: the two sides of the coin. Obes Facts. 2020 Jul;1-9.

2 Frühbeck G, Baker JL, Busetto L, Dicker D, Goossens GH, Halford JC, et al. European Association for the Study of Obesity Position Statement on the Global COVID-19 Pandemic. Obes Facts. 2020;13(2):292-6.

3 Albashir AA. The potential impacts of obesity on COVID-19. Clin Med (Lond). 2020 Jul;20(4):e109-13.

4 Docherty AB, Harrison EM, Green CA, Hardwick HE, Pius R, Norman L, et al. Features of 20133 UK patients in hospital with COVID-19 using the ISARIC WHO Clinical Characterisation Protocol: prospective observational cohort study. BMJ. 2020;369:m1985.

5 Williamson E, Walker AJ, Bhaskaran KJ, Bacon S, Bates C, Morton CE, et al. OpenSAFELY: factors associated with COVID-19-related hospital death in the linked electronic health records of 17 million adult NHS patients. medRxiv. 2020 May 07. https://doi.org/10.1101/2020.05.06.20092999

6 Lighter J, Phillips M, Hochman S, Sterling S, Johnson D, Francois F, et al. Obesity in patients younger than 60 years is a risk factor for COVID-19 hospital admission. Clin Infect Dis. 2020 Jul;71(15):896-7.

7 Rubino F, Cohen RV, Mingrone G, le Roux CW, Mechanick JI, Arterburn DE, et al. Bariatric and metabolic surgery during and after the COVID-19 pandemic: DSS recommendations for management of surgical candidates and postoperative patients and prioritisation of access to surgery. Lancet Diabetes Endocrinol. 2020 Jul; 8(7):640-8. 